\title{
Diagnostic Confusion Caused by a Giant Appendicolith: A Case Report
}

\author{
Kaushik Kumara, b, David Lewis $^{\mathrm{a}}$
}

\begin{abstract}
Acute appendicitis is one of the most common surgical emergencies worldwide. The diagnosis is usually a clinical one, supported by laboratory and radiological investigations as required. However, atypical clinical presentation and imaging can cause diagnostic confusion. Possibility of appendicolith in imaging in presence of abdominal pain and no urological pathology warrant diagnostic laparoscopy to rule out appendicitis, as appendicoliths are associated with complicated appendicitis particularly when they are large. We report a case of a 24-year-old male patient with clinical presentation suggestive of urological problem, and the imaging showed unusual large opacities in right lower abdomen with possibility of foreign body in small bowel or large fecalith. A diagnostic laparoscopy was performed where perforated appendix with large fecalith was confirmed.
\end{abstract}

Keywords: Appendicitis; Appendicolith; Fecalith; Giant appendicolith

\section{Introduction}

Acute appendicitis is one of the common surgical emergencies worldwide affecting approximately $7 \%$ of the general population in a lifetime and accounts for about $1 \%$ of all surgical operations [1]. Usually the diagnosis is made clinically with classic history and examination findings, supported by laboratory investigations. The ultrasound or CT scan is used when there is diagnostic uncertainty or to exclude other associated pathology.

However, sometimes the clinical presentation is not with classic features and imaging does not confirm clear diagno-

Manuscript accepted for publication January 05, 2015

${ }^{a}$ General Surgery Department, Hervey Bay Hospital, Urraween Road, QLD 4655, Australia

${ }^{b}$ Corresponding Author: Kaushik Kumar, General Surgery Department, Hervey Bay Hospital, 3, Kennedy Court, Urraween, QLD 4655, Australia. Email: drkaushik1977@gmail.com

doi: http://dx.doi.org/10.14740/jmc2032w sis and can lead to diagnostic confusion. Acute appendicitis can present with clinical features of urological problem and presence of large appendicolith in imaging can lead to further diagnostic dilemma.

\section{Case Report}

A 24-year-old male patient presented to the emergency department with 3 days history of abdominal pain described as intermittent colicky pain mainly in right lower quadrant and suprapubic region with some radiation of pain to right testes and right renal area. The patient also had few episode of nonbilious vomiting only on the first day of onset of the pain and mild dysuria in last 2 days. He denied any nausea, fever, anorexia, abdominal distension, change in bowel habits, hematuria or urethral discharge.

The examination of abdomen revealed soft non-distended abdomen with mild tenderness in right iliac fossa, hypogastrium and right renal angle. There was some voluntary guarding on deep palpation of lower abdomen. The groin examination did not show any hernia and external genitals were normal. There were normal bowel sounds on auscultation of abdomen. His temperature was $37.0^{\circ} \mathrm{C}$, heart rate was $104 / \mathrm{min}$ and blood pressure was $124 / 78 \mathrm{~mm} \mathrm{Hg}$. The blood reports were unremarkable (hemoglobin $158 \mathrm{mg} / \mathrm{dL}$, white blood cell count 10.6 , and normal renal and liver functions). A urine dipstick showed few leucocytes and few red blood cells but negative for nitrates. A CT KUB was organized with possible clinical diagnosis of ureteric stone that showed no renal calculi; however, there were two radio-opacities ( $2 \mathrm{~cm}$ and $5 \mathrm{~mm}$ size) in lower abdomen with possibility of foreign body in the small intestinal lumen or a large appendicolith (Fig. 1, 2). The appendix was not positively identified and there was no inflammatory stranding; however, there was some free fluid in pelvis. Patient denied any ingestion of tablets or foreign body.

Due to ongoing pain and tachycardia, a diagnostic laparoscopy was performed where a perforated appendix was noted in pelvic position with purulent fluids in pelvis. There was a giant fecalith in shaft of appendix with perforation over fecalith (Fig. 3 , 4). Laparoscopic appendicectomy was performed without much technical difficulty, peritoneal cavity was washed out with $2 \mathrm{~L}$ of normal saline and $15 \mathrm{~F}$ soft silicone drain was put in. The post-operative course was uneventful and patient 


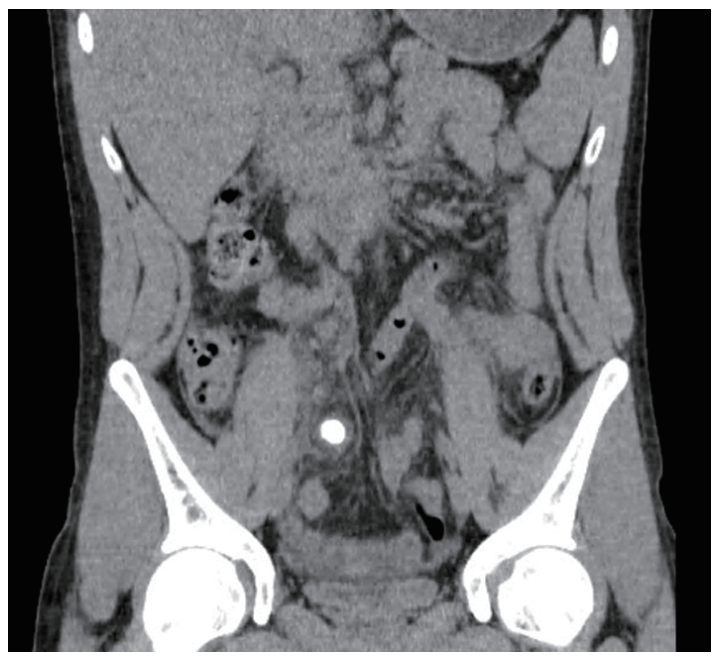

Figure 1. CT KUB coronal image with large radio-opacity in right lower quadrant.

was discharged on the third post-operative day after removal of drain.

\section{Discussion}

The classical features of acute appendicitis are initially poorly localized colicky abdominal pain due to obstruction of lumen (not severe as colicky pain of small bowel obstruction), which is usually first noticed in periumbilical region due to midgut origin of appendix. The pain is usually associated with anorexia, nausea and few episodes of vomiting. Anorexia is useful and constant clinical feature of appendicitis. With the progression of inflammation of appendix, irritation of parietal peritoneum occurs in right iliac fossa (RIF) and the pain becomes intense, constants and localizes to RIF. However, atypical presentations are also common with predominantly visceral or somatic poorly localized pain. In pelvic appendicitis, the somatic pain is usually absent and suprapubic discomfort and tenesmus are prominent features due to irritation of urinary bladder and rec-

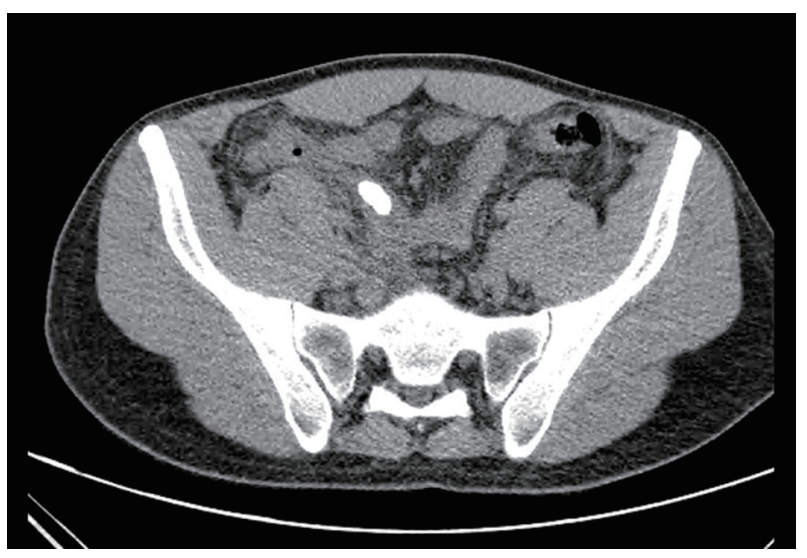

Figure 2. CT KUB axial image with large radio-opacity in right lower quadrant.

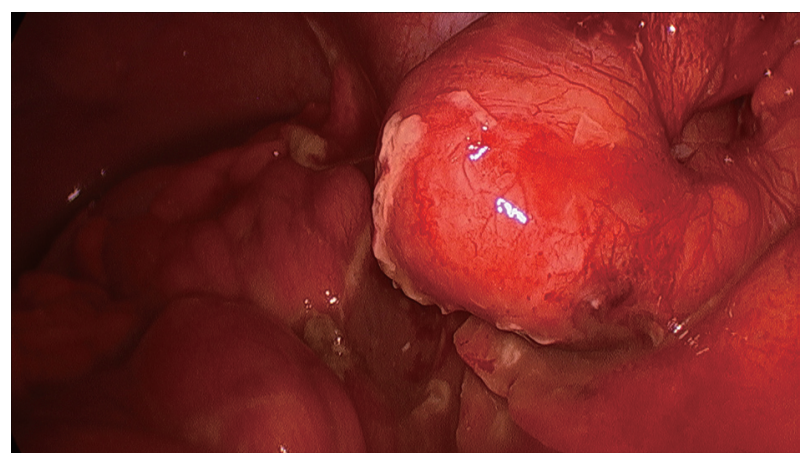

Figure 3. Laparoscopic images of appendix showing very large distended appendix with giant fecalith.

tum respectively. Cases have been reported where appendicitis has been presented with features suggestive of urological problems like the cases reported by Teke et al [1] and Bulent et al [2]. The ultrasound has been used since a long time as first method of imaging, while CT scan is used for adult population and when diagnostic confusion is there with high sensitivity and specificity (94\% and $95 \%$ respectively).

The most commonly accepted theory for appendicitis is that it results from obstruction followed by infection. The obstruction could be due to lymphoid hyperplasia, fecaliths, worms, stricture or tumor. Traditionally, fecaliths have been considered as main cause of appendicular obstruction; however, Singh et al in his study concluded against this [3].

Appendicolithiasis is a condition characterized by a concretion or calcified deposits in the vermiform appendix (It is also called appendiceal calculi, appendiceal enterolith, or coprolith). Most are fecaliths, which are tightly packed stool material, while, small minority are actual calculi, stone containing mineral deposits. Usually they are small $(<1 \mathrm{~cm})$ but few cases have been mentioned with giant appendicoliths $(>2 \mathrm{~cm}$ size $)$. They are considerable variation in prevalence of appendicoliths with appendicitis. Studies before 1970 have described high prevalence rate of $33-44 \%$, but large studies after 1970 have demonstrated low prevalence rate of $1.54-15 \%$ [3]. Most studied showed that they are more commonly associated with complicated appendicitis with perforation and abscess formation (prevalence of 39.4-50\%).

They are more common in male patients than female and

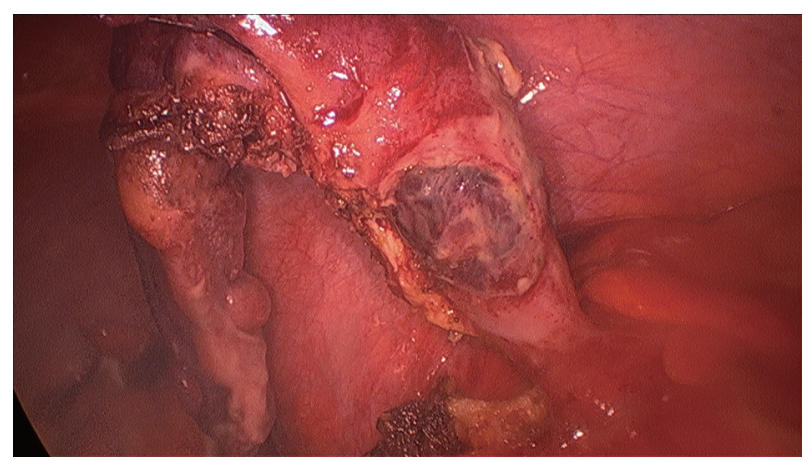

Figure 4. Laparoscopic image of appendicular perforation site with underlying large fecalith. 
in retrocecal appendix position [4]. Due to increasing use of imaging including CT scan, they are more frequently detected. The reported sensitivity of CT scan for detection of appendicoliths is only $53.1 \%$. They appear as laminated bodies with gas in center or homogenous opacity in CT scan depending up on level of calcification.

When they are detected in presence of abdominal pain, there is $90 \%$ probability of acute appendicitis and $50 \%$ higher risk of appendiceal perforation and abscess formation. The significance of incidental finding of appendicolith in imaging without abdominal pain or CT scan findings of appendicitis is still debatable. Rabinowitz et al in his study of 75 patients with appendicoliths concluded that appendicoliths may be associated with increased risk of appendicitis but is not a pure indication of appendicectomy [5]. Grimes et al in her study concluded that appendicoliths may be associated with recurrent RIF pain and routine removal of normal looking appendix during diagnostic laparoscopy for RIF pain will pick up 10$15 \%$ patients that contains fecaliths and offers potential cure, prevent recurrent admission and economic benefit as well [6].

In the present case, the presenting features and findings were more of urological problem: colicky abdominal pain with radiation to right testes and mild tenderness in right renal area with presence of microscopic hematuria and few leucocytes and normal white cell count. However, these findings are sometime associated with perforated appendix. Microscopic hematuria and leucocytes could be reactionary inflammation of bladder or ureter. The non-contrast CT scan was not suggestive of urological problem. There were no obvious signs of appendicitis in plain CT and due to large size of appendicolith there was diagnostic confusion. Due to ongoing pain and tachycardia and possibility of a large appendix with appendicolith, diagnostic laparoscopy was performed.

\section{Conclusion}

Although acute appendicitis is usually a clinical diagnosis, atypical clinical presentation and pathological and radiological investigations can cause diagnostic confusion. Possibility of appendicolith in imaging in presence of abdominal pain and no urological pathology warrant diagnostic laparoscopy to rule out appendicitis as appendicoliths are associated with compli- cated appendicitis particularly when they are large.

\section{Grant Support}

None.

\section{Conflict of Interest}

Authors declare no conflict of interest regarding this article.

\section{Author Contributions}

Dr Lewis performed surgery and Dr Kumar assisted him. Patient's data and manuscript preparation was done by $\mathrm{Dr} \mathrm{Ku}-$ mar. Both authors have reviewed and approved final version of manuscript.

\section{References}

1. Teke Z, Kabay B, Erbis H, Tuncay OL. Appendicolithiasis causing diagnostic dilemma: a rare cause of acute appendicitis (report of a case). Ulus Travma Acil Cerrahi Derg. 2008;14(4):323-325.

2. Kaya B, Eris C. Different clinical presentation of appendicolithiasis. The report of three cases and review of the literature. Clin Med Insights Pathol. 2011;4:1-4.

3. Singh JP, Mariadason JG. Role of the faecolith in modernday appendicitis. Ann R Coll Surg Engl. 2013;95(1):4851 .

4. Nitecki S, Karmeli R, Sarr MG. Appendiceal calculi and fecaliths as indications for appendectomy. Surg Gynecol Obstet. 1990;171(3):185-188.

5. Rabinowitz CB, Egglin TK, Beland MD, Mayo-Smith WW. Outcomes in 74 patients with an appendicolith who did not undergo surgery: is follow-up imaging necessary? Emerg Radiol. 2007;14(3):161-165.

6. Grimes C, Chin D, Bailey C, Gergely S, Harris A. Appendiceal faecaliths are associated with right iliac fossa pain. Ann R Coll Surg Engl. 2010;92(1):61-64. 